\title{
Phase-difference measurement in iVLBI and application in the measurement of lunar rotation
}

\author{
Ming Chen ${ }^{1}$, Nobuyuki Kawano ${ }^{1}$, Kun Shang ${ }^{1}$, Jing Sun $^{1}$, Qinghui Liu ${ }^{1}$, Fuyuhiko Kikuchi $^{2}$, and Jinsong Ping ${ }^{1}$ \\ ${ }^{1}$ Shanghai Astronomical Observatory, Shanghai, China, 200030 \\ ${ }^{2}$ National Astronomical Observatory, Mizusawa 023-0861, Japan
}

(Received May 12, 2010; Revised December 9, 2010; Accepted February 7, 2011; Online published June 21, 2011)

\begin{abstract}
Radio waves emitted from two or more landing units on the lunar surface are received by an antenna at the Earth station, and the range differences between these landing units are measured with an error of several millimeters. The phase differences between the oscillators of these landing units are monitored via an orbiter that orbits around the Moon. We have developed a simple roundtrip method to obtain these phase differences and also propose a method of calibrating the system delay on the ground. In order to observe the rotation of the Moon and monitor the phase differences effectively, we designed the position of the landing units and the orbit of the orbiter. Further, we concurrently analyzed the characteristics of the common view period. The error of the system was analyzed and to have a high accuracy. The results show that inverse VLBI technology can be used to measure the rotation of the Moon and new scienti c results can be obtained.
\end{abstract}

Key words: iVLBI, lunar rotation, roundtrip method, NBV, orbit design, common view period.

\section{Introduction}

Both the conventional Very Long Baseline Interferometry (VLBI) technique and laser ranging have certain limitations in their ability for ground-based geodetic observation of the Moon, with an accuracy reaching up to a few centimeters. Regarding laser ranging technology, the observation period is limited as it cannot be used to observe the Moon during a full or new Moon. In comparison, the VLBI technique is associated with an error caused by the propagation media. The instrument cannot be corrected with high accuracy and, therefore, the accuracy of the delay is limited to almost 0.1 ns. Kawano et al. (1999) proposed that the tides and rotational variations of the Moon or planets could be observed with greater accuracy using a new method, called the "Inverse VLBI (iVLBI)". This method enables geodetic measurement of the Moon with an error of a few millimeters, which is the same as that for observations from the Earth. Following further development of the concept and design of the iVLBI, as well as demonstration of its applications in science, the two-way method was recommended for synchronizing the reference frequency that was generated by the landing units (Kawano et al., 2009). A different direct ranging method was proposed to monitor the standard frequencies (Kawano et al., 2010). The iVLBI technology was also selected as a candidate research project in SELENE 2. We are aware that the time-transfer and ranging experiment between the ground and a satellite has been performed in the ETS-VIII experiment (Takahashi et al., 2008). Precise time-transfer is a reference technique

Copyright (c) The Society of Geomagnetism and Earth, Planetary and Space Sciences (SGEPSS); The Seismological Society of Japan; The Volcanological Society of Japan; The Geodetic Society of Japan; The Japanese Society for Planetary Sciences; TERRAPUB.

doi:10.5047/eps.2011.02.005 applied in order to realize the iVLBI system. The phase difference between the signals of the two landing units is an essential parameter and needs to be measured as it directly in uences the accuracy of the result. In this article, we propose a solution to the phase-difference measurement.

This article comprises eight sections. Section 1 is the introduction; Section 2 discusses the principle of iVLBI; Section 3 describes the phase-difference measurement of the two landers and its accuracy; examples of frequency selection and link budget are shown in Section 4; Section 5 presents a design of the position of the landers on the lunar surface and the orbit of the orbiter; Section 6 includes an analysis of the accuracy of the delay derived by the iVLBI observation; in Section 7, we demonstrate the possible application of the iVLBI technology in scienti c research. The article closes with the conclusion and discussion in Section 8 .

\section{Principle of iVLBI}

The observation system of the iVLBI consists of three sub-systems. These include (1) two or more landing units on the Moon, (2) an orbiter orbiting around the Moon, and (3) a ground station. Phase differences among radio waves that are transmitted on the Moon are monitored through the orbiter. An antenna/receiver on the ground station receives radio waves from all the landing units on the Moon simultaneously, and phase differences can be measured among the radio waves received at the ground station. The range differences among the landing units and the antenna of the ground station can be obtained after correcting for the phase differences among radio waves transmitted from the landing units on the Moon. Tides and rotational variations of the Moon can be detected with a higher accuracy using the iVLBI system than is currently possible with VLBI obser- 


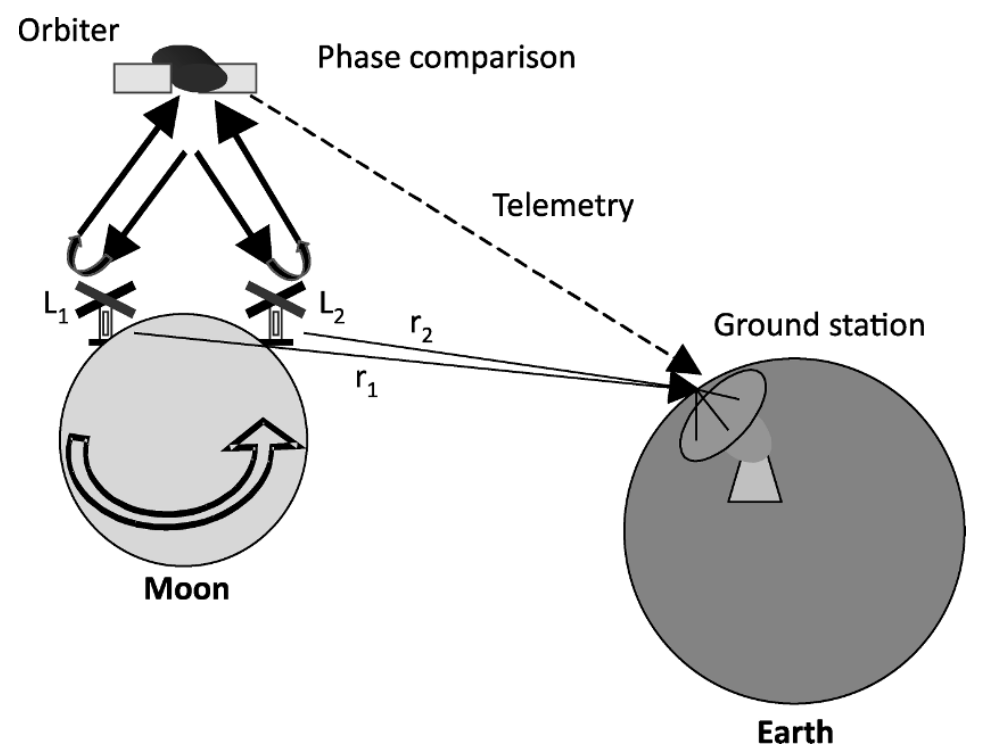

Fig. 1. Concept of inverse VLBI.

vation or lunar laser ranging on the Earth. The accuracy of the range difference between two landing units and the antenna on the Earth depends on the measurement error of the phase difference between the two landing units.

The data obtained by this system are analyzed as a combination of a pair of landing units, an orbiter, and an antenna of a ground station. The concept of the system described here is depicted in Fig. 1. The Moon is suf ciently distant from the Earth that both the landing units and the orbiter are within the main beam of the antenna at the ground station. Therefore, radio waves transmitted from the two landing units are received simultaneously by the antenna of the ground station. The measured phase difference between the two radio waves includes the phase difference of the two units. Changes in the phase difference are monitored by the orbiter, and the results are sent to the ground station via a telemetry signal. The phase changes of the oscillators in the two landing units are then subtracted from the measured phase difference between the two radio waves received at the ground station. Thus, the range difference $r_{2}-r_{1}$ in Fig. 1 can be obtained, and this can be used to solve the libration parameters of the Moon.

\section{Phase-Difference Measurements of the Two Landers}

3.1 Phase-difference measurements between a roundtrip signal without carrier recovery and return signal in a satellite

A block diagram of the system depicting a simple roundtrip method without carrier recovery is shown in Fig. 2. The delay model generated by this system is shown in Fig. 3.

It is assumed that all of the frequencies transmitted from a satellite are produced by the same frequency standard, and the frequency is assumed to be $f_{\mathrm{SAT}}^{0}$, with phase $\Phi_{\mathrm{SAT} 0}$. Each frequency signal that is transmitted from the antenna of the satellite has its own initial phase $\phi_{\mathrm{SF} 0}^{i}(i=1,2,3$ : indicating frequency). The phase of the signal transmitted from the satellite is given by

$$
\phi_{\mathrm{SF}}^{i}=2 \pi f^{i} t-2 \pi f_{\mathrm{SAT}}^{0} \cdot \tau_{\mathrm{STF}}^{i}-2 \pi f^{i} \cdot \tau_{\mathrm{SF}}^{i}+\sigma_{\phi}^{i}+\phi_{\mathrm{SF} 0}^{i}
$$

where $\tau_{\mathrm{STF}}^{i}$ is the delay from the frequency standard to the frequency synthesizer for $f^{i} ; \tau_{\mathrm{SF}}^{i}$ is the delay from the synthesizer to the antenna of the satellite; $\phi_{\mathrm{SF} 0}^{i}$ correlates with the initial phase of the frequency standard of the satellite. This standard is dependent on how the transmitting signal is produced, and it is dif cult to accurately judge the phase. For this reason, $\phi_{\mathrm{SF} 0}^{i}$ is taken as an unknown constant, and $\sigma_{\phi}^{i}$ is the phase variation of a carrier signal generated by the frequency standard. The variation is almost nulli ed in the difference between the roundtrip and return signals, although the phase variation within the period of the roundtrip time persists. This phase variation is discussed later in the article.

The transmitted signals from the antenna of the satellite reach a landing unit after a propagation time $R_{\mathrm{S}-\mathrm{L} 1} / c$. Taking into account the delay $\tau_{\mathrm{L} 1 \mathrm{~F}}^{i}$ from the antenna of lander 1 to the mixer, the phase of the signal at the mixer is derived by

$$
\begin{aligned}
\phi_{\mathrm{L} 1 \mathrm{~F}}^{i}= & 2 \pi f^{i} t-2 \pi f_{\mathrm{SAT}}^{0} \cdot \tau_{\mathrm{STF}}^{i}-2 \pi f^{i} \cdot \tau_{\mathrm{SF}}^{i} \\
& -2 \pi f^{i} \cdot\left(R_{\mathrm{S}-\mathrm{L} 1} / c\right)-2 \pi f^{i} \cdot \tau_{\mathrm{L} 1 \mathrm{~F}}^{i} \\
& +\sigma_{\phi}^{i}+\phi_{\mathrm{SF} 0}^{i}
\end{aligned}
$$

In the mixer, the frequency signal $f_{\mathrm{L} 1}^{0}$ with initial phase $\Phi_{\mathrm{L} 10}$ is mixed with the received signal, and the frequency changes to $f^{i}-f_{\mathrm{L} 1}^{0}$. Mixing the frequency signal $f_{\mathrm{L} 1}^{0}$ with the received signal at lander 1 is the simplest method for reserving the initial phase $\Phi_{\mathrm{L} 10}$ in the received signal at the satellite. The phase variation of the frequency standard of lander 1 is included in $\Phi_{\mathrm{L} 10}$. The phase of the transmitted signal from the antenna of lander 1 is obtained by

$$
\begin{aligned}
\phi_{\mathrm{L} 1 \mathrm{~B}}^{i}= & 2 \pi\left(f^{i}-f_{\mathrm{L} 1}^{0}\right) t-2 \pi f_{\mathrm{SAT}}^{0} \cdot \tau_{\mathrm{STF}}^{i}-2 \pi f^{i} \cdot \tau_{\mathrm{SF}}^{i} \\
& -2 \pi f^{i} \cdot\left(R_{\mathrm{S}-\mathrm{L} 1} / c\right)-2 \pi f^{i} \cdot \tau_{\mathrm{L} 1 \mathrm{~F}}^{i}
\end{aligned}
$$




\section{Block diagram of the roundtrip method}

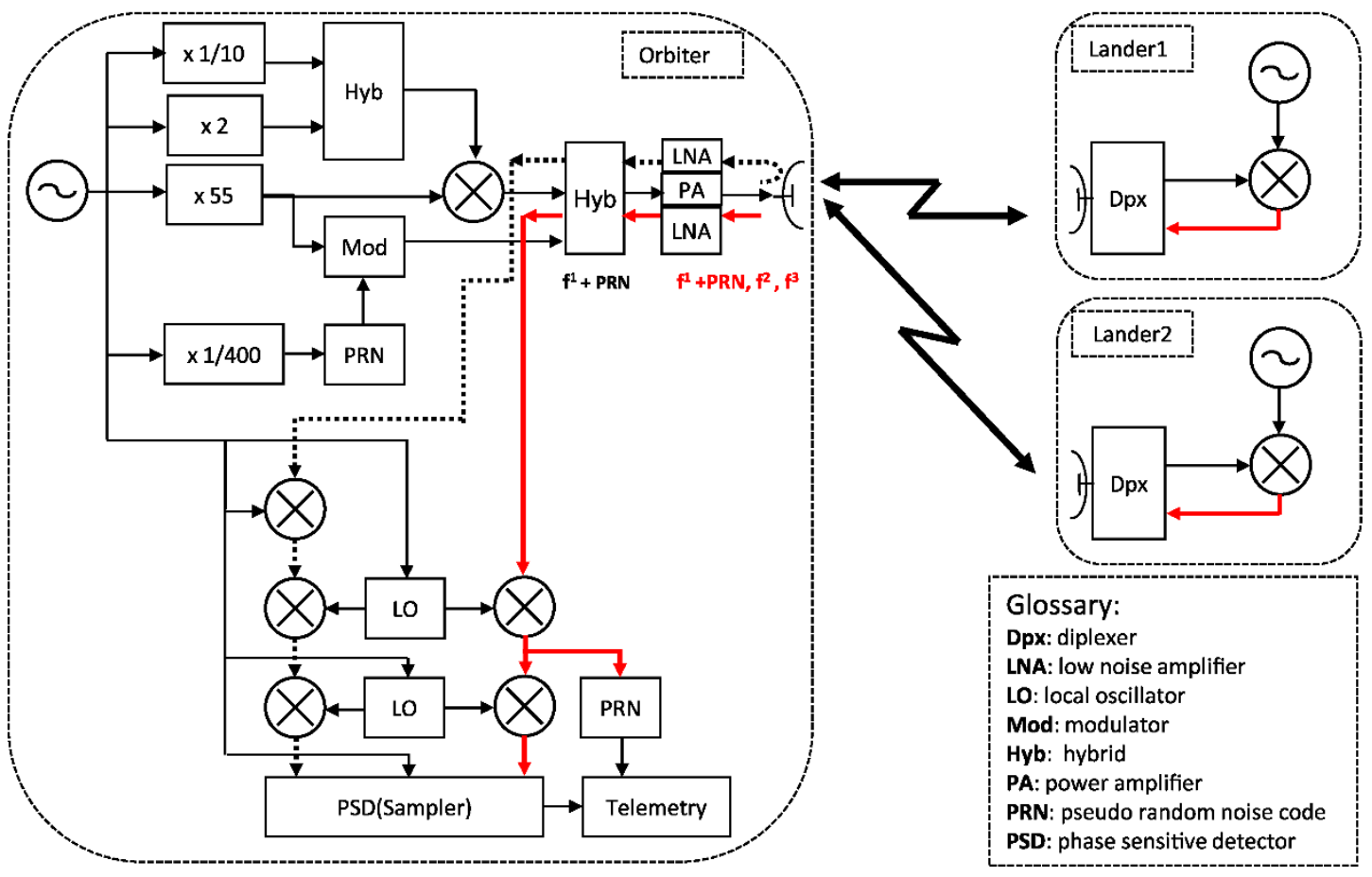

Fig. 2. A simple roundtrip method.

Delay model of the roundtrip method

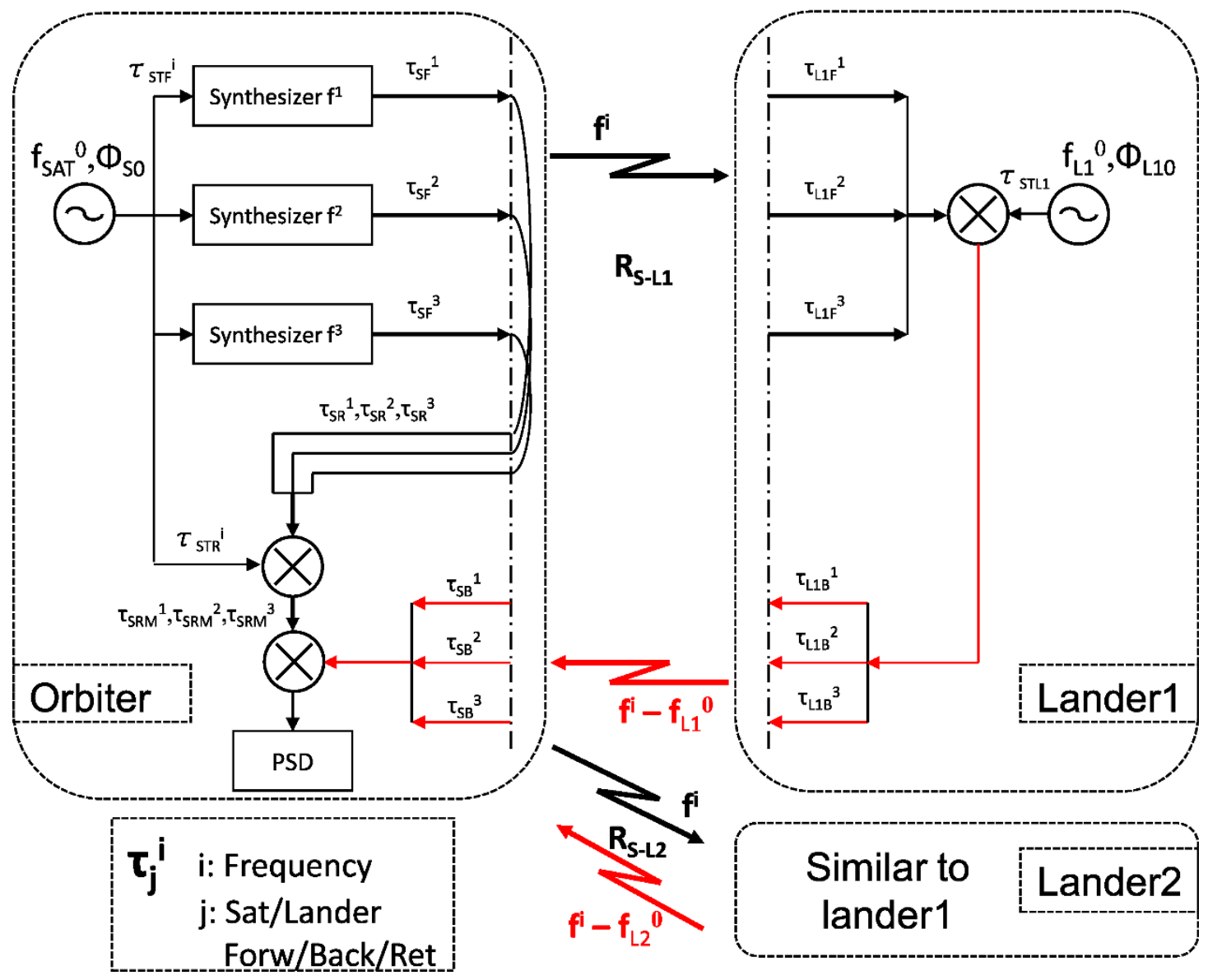

Fig. 3. The delay model of the simple roundtrip method. 


$$
\begin{aligned}
& -2 \pi\left(f^{i}-f_{L 1}^{0}\right) \cdot \tau_{L 1 B}^{i}+2 \pi f_{L 1}^{0} \cdot \tau_{\mathrm{STL} 1} \\
& +\sigma_{\phi}^{i}+\phi_{\mathrm{SF} 0}^{i}-\Phi_{\mathrm{L} 10}
\end{aligned}
$$

where $\tau_{\mathrm{STL} 1}$ is the delay from the frequency standard to the mixer of lander 1 , and $\tau_{\mathrm{L} 1 \mathrm{~B}}^{i}$ is the delay from the mixer to the antenna in lander 1 .

The transmitted signals from lander 1 reach the satellite after a propagation time of $R_{\mathrm{S}-\mathrm{L} 1}^{\prime} / C$. The $R_{\mathrm{S}-\mathrm{L} 1}^{\prime}$ is slightly different from the $R_{\mathrm{S}-\mathrm{L} 1}$ because the satellite and lander 1 move during the propagation time $R_{\mathrm{S}-\mathrm{L} 1} / C$. However, the difference is very small and can be easily corrected by the rough predicted orbit of the satellite, such that $R_{\mathrm{S}-\mathrm{L} 1}^{\prime}$ is presumed to be equal to $R_{\mathrm{S}-\mathrm{L} 1}$ here. The phase of the received signal at the antenna of the satellite is expressed as

$$
\begin{aligned}
\phi_{\mathrm{SB}}^{i}= & 2 \pi\left(f^{i}-f_{\mathrm{L} 1}^{0}\right) t-2 \pi f_{\mathrm{SAT}}^{0} \cdot \tau_{\mathrm{STF}}^{i}-2 \pi f^{i} \cdot \tau_{\mathrm{SF}}^{i} \\
& -2 \pi f^{i} \cdot\left(R_{\mathrm{S}-\mathrm{L} 1} / c\right)-2 \pi f^{i} \cdot \tau_{\mathrm{L} 1 \mathrm{~F}}^{i} \\
& -2 \pi\left(f^{i}-f_{\mathrm{L} 1}^{0}\right) \cdot \tau_{\mathrm{L} 1 \mathrm{~B}}^{i}-2 \pi\left(f^{i}-f_{\mathrm{L} 1}^{0}\right) \cdot\left(R_{\mathrm{S}-\mathrm{L} 1} / c\right) \\
& +2 \pi f_{\mathrm{L} 1}^{0} \cdot \tau_{\mathrm{STL} 1}+\sigma_{\phi}^{i}+\phi_{\mathrm{SF} 0}^{i}-\Phi_{\mathrm{L} 10}
\end{aligned}
$$

The signal received at the satellite is called the roundtrip signal. Taking into account the delay $\tau_{\mathrm{SB}}^{i}$ from the antenna to the mixer of the satellite, the phase of the roundtrip signal can be expressed as,

$$
\begin{aligned}
\phi_{\mathrm{PSD}}^{i}= & 2 \pi\left(f^{i}-f_{\mathrm{L} 1}^{0}\right) t-2 \pi f_{\mathrm{SAT}}^{0} \cdot \tau_{\mathrm{STF}}^{i}-2 \pi f^{i} \cdot \tau_{\mathrm{SF}}^{i} \\
& -2 \pi f^{i} \cdot\left(R_{\mathrm{S}-\mathrm{L} 1} / c\right)-2 \pi f^{i} \cdot \tau_{\mathrm{L} 1 \mathrm{~F}}^{i} \\
& -2 \pi\left(f^{i}-f_{\mathrm{L} 1}^{0}\right) \cdot \tau_{\mathrm{L} 1 \mathrm{~B}}^{i}-2 \pi\left(f^{i}-f_{\mathrm{L} 1}^{0}\right) \cdot\left(R_{\mathrm{S}-\mathrm{L} 1} / c\right) \\
& -2 \pi\left(f^{i}-f_{\mathrm{L} 1}^{0}\right) \cdot \tau_{\mathrm{SB}}^{i}+2 \pi f_{\mathrm{L} 1}^{0} \cdot \tau_{\mathrm{STL} 1} \\
& +\sigma_{\phi}^{i}+\phi_{\mathrm{SF} 0}^{i}-\Phi_{\mathrm{L} 10}
\end{aligned}
$$

The signal which returns directly from the antenna in the satellite is named the return signal and reaches the mixer after a delay $\tau_{\mathrm{SR}}^{i}$. The phase of the return signal in the satellite at the input of the mixer is expressed as

$$
\phi_{\mathrm{SR}}^{i}=2 \pi f^{i} t-2 \pi f_{\mathrm{SAT}}^{0} \cdot \tau_{\mathrm{STF}}^{i}-2 \pi f^{i} \cdot \tau_{\mathrm{SF}}^{i}-2 \pi f^{i} \cdot \tau_{\mathrm{SR}}^{i}+\sigma^{\prime \prime}{ }_{\phi}+\phi_{\mathrm{SF} 0}^{i}
$$

where $\sigma_{\phi}^{\prime i}$ is the phase variation of the carrier signal and is caused by the instability of the frequency standard in the satellite. This is not the same as the $\sigma_{\phi}^{i}$, because $\sigma_{\phi}^{\prime i}$ is the phase variation that is delayed by the roundtrip time.

When the return signal is mixed with the local frequency standard $f_{\mathrm{SAT}}^{0}$, a phase can be obtained as

$$
\begin{aligned}
\phi_{\mathrm{PSDR}}^{i}= & 2 \pi\left(f^{i}-f_{\mathrm{SAT}}^{0}\right) t-2 \pi f_{\mathrm{SAT}}^{0} \cdot \tau_{\mathrm{STF}}^{i}-2 \pi f^{i} \cdot \tau_{\mathrm{SF}}^{i} \\
& -2 \pi f^{i} \cdot \tau_{\mathrm{SR}}^{i}+2 \pi f_{\mathrm{SAT}}^{0} \cdot \tau_{\mathrm{STR}}^{i} \\
& -2 \pi\left(f^{i}-f_{\mathrm{SAT}}^{0}\right) \cdot \tau_{\mathrm{SRM}}^{i}+\phi_{\mathrm{SF} 0}^{i} \\
& +\sigma_{\phi}^{\prime i}-\Phi_{\mathrm{S} 0}
\end{aligned}
$$

where the phase variation caused by the instability of the frequency standard in the satellite is included in $\Phi_{\mathrm{s} 0}$ (the phase variation of $f_{\mathrm{SAT}}^{0}$ is very small compared with ${\sigma_{\phi}^{\prime i}}_{\phi}$ ), and $\tau_{\mathrm{STR}}^{i}$ is the delay from the frequency standard to the mixer in the satellite.

After mixing with the local frequency standard, the return signal is compared with the roundtrip signal in the satellite. The phase difference obtained as the result of phase comparison is

$$
\begin{aligned}
\Delta \phi_{\mathrm{PSD}}^{i}= & \phi_{\mathrm{PSD}}^{i}-\phi_{\mathrm{PSDR}}^{i} \\
= & -2 \pi \cdot \delta f_{\mathrm{L} 1 \mathrm{SAT}} \cdot t \\
& -2 \pi f^{i} \cdot\left(\tau_{\mathrm{L} 1 \mathrm{~F}}^{i}-\tau_{\mathrm{SR}}^{i}\right) \\
& -2 \pi\left(f^{i}-f_{\mathrm{SAT}}^{0}\right) \cdot\left(\tau_{\mathrm{L} 1 \mathrm{~B}}^{i}+\tau_{\mathrm{SB}}^{i}-\tau_{\mathrm{SRM}}^{i}\right) \\
& +2 \pi \cdot \delta f_{\mathrm{L} 1 \mathrm{SAT}} \cdot \tau_{\mathrm{L} 1 \mathrm{~B}}^{i}+2 \pi \cdot \delta f_{\mathrm{L} 1 \mathrm{SAT}} \cdot \tau_{\mathrm{SB}}^{i} \\
& -2 \pi f^{i} \cdot\left(R_{\mathrm{S}-\mathrm{L} 1} / c\right) \\
& -2 \pi\left(f^{i}-f_{\mathrm{SAT}}^{0}\right) \cdot\left(R_{\mathrm{S}-\mathrm{L} 1} / c\right) \\
& +2 \pi \cdot \delta f_{\mathrm{L} 1 \mathrm{SAT}} \cdot\left(R_{\mathrm{S}-\mathrm{L} 1 / c}\right) \\
& +2 \pi f_{\mathrm{SAT}}^{0} \cdot\left(\tau_{\mathrm{STL} 1}-\tau_{\mathrm{STR}}^{i}\right)-2 \pi \cdot \delta f_{\mathrm{L} 1 \mathrm{SAT}} \cdot \tau_{\mathrm{STL} 1} \\
& -\left(\Phi_{\mathrm{L} 10}-\Phi_{\mathrm{S} 0}\right)+\delta \sigma_{\phi}^{i}
\end{aligned}
$$

where $\delta f_{\mathrm{L} 1 \mathrm{SAT}}=f_{\mathrm{L} 1}^{0}-f_{\mathrm{SAT}}^{0}, \delta \sigma_{\phi}^{i}=\sigma_{\phi}^{i}-{\sigma^{\prime}{ }_{\phi}}^{i}$. The frequency standard is usually designed such that $\delta f_{\text {L1SAT }}$ is small enough. The values $\delta f_{\mathrm{L} 1 \mathrm{SAT}} \cdot \tau_{\mathrm{L} 1 \mathrm{~B}}^{i}, \delta f_{\mathrm{L} 1 \mathrm{SAT}} \dot{\tau}_{\mathrm{SB}}^{i}$, $\delta f_{\mathrm{L} 1 \mathrm{SAT}} \cdot \tau_{\mathrm{STL} 1}$ and $\delta f_{\mathrm{L} 1 \mathrm{SAT}} \cdot\left(R_{\mathrm{S}-\mathrm{L} 1} / c\right)$ are minor and can be ignored as they are of no signi cance. The term of the $\delta \sigma_{\phi}^{i}$ is also minimal and is neglected hereafter. For a long period, the phase difference of $\delta f_{\mathrm{L} 1 \mathrm{SAT}}$ may drift to a certain extent, and the phase value of $2 \pi \cdot \delta f_{\text {L1SAT }} \cdot t$ cannot be omitted. Further, we provide a method for the estimation of this value. By using the estimated phase value, the differential phase value can be modi ed to remove the phase caused by $\delta f_{\mathrm{L} 1 \mathrm{SAT}}$. Substituting $\left(f^{i}-f_{\mathrm{SAT}}^{0}\right)$ to $r^{i} f^{i}, \Delta \phi_{\mathrm{PSD}}^{i}$ can be rewritten as

$$
\begin{aligned}
\Delta \phi_{\mathrm{PSD}}^{i}= & -2 \pi f^{i} \cdot\left[\left(\tau_{\mathrm{L} 1 \mathrm{~F}}^{i}-\tau_{\mathrm{SR}}^{i}\right)+r^{i} \cdot\left(\tau_{\mathrm{L} 1 \mathrm{~B}}^{i}+\tau_{\mathrm{SB}}^{i}-\tau_{\mathrm{SRM}}^{i}\right)\right] \\
& -2 \pi f^{i} \cdot\left(1+r^{i}\right) \cdot\left(R_{\mathrm{S}-\mathrm{L} 1} / c\right) \\
& +2 \pi f_{\mathrm{SAT}}^{0} \cdot\left(\tau_{\mathrm{STL} 1}-\tau_{\mathrm{STR}}^{i}\right) \\
& -\left(\Phi_{\mathrm{L} 10}-\Phi_{\mathrm{S} 0}\right)
\end{aligned}
$$

The phase of $2 \pi f_{\mathrm{SAT}}^{0}\left(\tau_{\mathrm{STL} 1}-\tau_{\mathrm{STR}}^{i}\right)$ is proportional to the lower frequency $f_{\mathrm{SAT}}^{0}$ instead of the RF (radio frequency).

Finally, the measured phase difference can be obtained as

$$
\begin{aligned}
\Delta \phi_{\mathrm{PSD}}^{i}= & -2 \pi f^{i} \cdot \tau_{\mathrm{TOTAL}}^{i}-2 \pi f^{i} \cdot\left(1+r^{i}\right) \cdot\left(R_{\mathrm{S}-\mathrm{L} 1} / c\right) \\
& +2 \pi f_{\mathrm{SAT}}^{0} \cdot \delta \tau_{\mathrm{ST}}-\left(\Phi_{\mathrm{L} 10}-\Phi_{\mathrm{S} 0}\right)
\end{aligned}
$$

where, $\tau_{\mathrm{TOTAL}}^{i}=\left(\tau_{\mathrm{L} 1 \mathrm{~F}}^{i}-\tau_{\mathrm{SR}}^{i}\right)+r^{i} \cdot\left(\tau_{\mathrm{L} 1 \mathrm{~B}}^{i}+\tau_{\mathrm{SB}}^{i}-\tau_{\mathrm{SRM}}^{i}\right)$, $\delta \tau_{\mathrm{ST}}=\tau_{\mathrm{STL} 1}-\tau_{\mathrm{STR}}^{i}$.

Assuming that the $2 \pi f_{\mathrm{SAT}}^{0} \delta \tau_{\mathrm{ST}}$ is small and can be measured separately and corrected prior to the launch, Eq. (8) can be expressed as,

$$
\begin{aligned}
\Delta \phi_{\mathrm{PSD}}^{i}= & -2 \pi f^{i} \cdot \tau_{\mathrm{TOTAL}}^{i}-2 \pi f^{i} \cdot\left(1+r^{i}\right) \cdot\left(R_{\mathrm{S}-\mathrm{L} 1} / c\right) \\
& -\left(\Phi_{\mathrm{L} 10}-\Phi_{\mathrm{S} 0}\right)
\end{aligned}
$$

$\tau_{\text {TOTAL }}^{i}$ is different for the variable frequency $f^{i}$, but remains constant as long as the temperature of this system 


\section{Calibration on the ground}

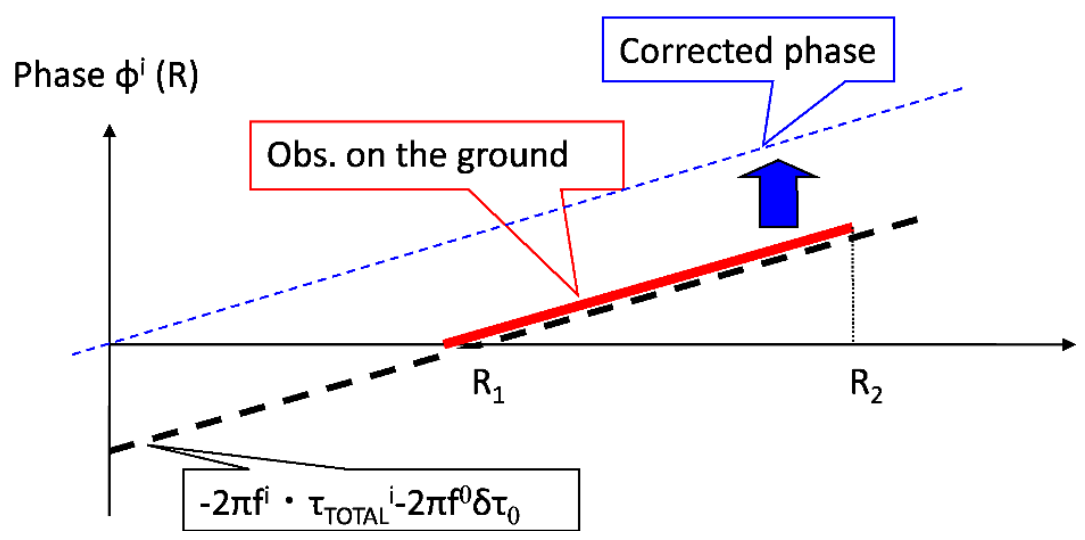

Fig. 4. Concept of the calibration on the ground.

and the voltage of power supply do not vary. The entire delay would be of a few nanoseconds.

For lander 2, Eq. (9) can also be derived; however, the delays for lander 2 , which are $\tau_{\mathrm{L} 2 \mathrm{~B}}^{i}$ and $\tau_{\mathrm{L} 2 \mathrm{~F}}^{i}$, are different from $\tau_{\mathrm{L} 1 \mathrm{~B}}^{i}$ and $\tau_{\mathrm{L} 1 \mathrm{~F}}^{i}$ and, therefore, $\tau_{\mathrm{TOTAL}}^{i}$ and $\left(\Phi_{\mathrm{L} 10}-\Phi_{\mathrm{s} 0}\right)$ cannot be estimated separately from Eq. (9). In order to determine $\tau_{\text {TOTAL }}^{i}$, calibration on the ground is required.

There is a method, in principle, to determine the $2 \pi f^{i}$. $\tau_{\text {TOTAL }}^{i}$ separately, and this is called a phase calibrator. A pulse is inserted at the front end and produces many frequency components after a band-pass filter. The phases are measured by the tone signal that is produced by the pulse at near-signal frequencies, and the delay produced in the instruments can also be calculated. This instrument is, of course, kept at a constant temperature. However, in reality, it is rather difficult to determine $2 \pi f^{i} \cdot \tau_{\text {TOTAL }}^{i}$ separately with high accuracy, although the instrumental total delay variation can be monitored to a certain extent. This could help predict the $2 \pi f^{i} \cdot \tau_{\text {TOTAL }}^{i}$ separately.

3.2 Calibration of the instrumental delay on the ground by using a common frequency standard

We consider a method to calculate $2 \pi f^{i} \cdot \tau_{\mathrm{TOTAL}}^{i}+\left(\Phi_{\mathrm{L} 10}-\right.$ $\left.\Phi_{\mathrm{s} 0}\right)$ in Eq. (8). If the phase with known $R_{\mathrm{S}-\mathrm{L} 1}$ on the ground is measured before launch, $2 \pi f^{i} \cdot \tau_{\text {TOTAL }}^{i}+\left(\Phi_{\mathrm{L} 10}-\right.$ $\left.\Phi_{\mathrm{s} 0}\right)$ can be obtained. The standard frequency signal is often used for both units of the lander and the satellite. The delay of each cable supplying the frequency standard is, of course, well calibrated. Then, $2 \pi f^{0} \delta \tau_{0}-\left(\Phi_{\mathrm{L} 10}-\Phi_{\mathrm{s} 0}\right)$ can be considered a known constant, $\delta \tau_{0}$ the delay difference of the two cables, and $f^{0}$ the frequency standard of the satellite and the lander. The $\left(\Phi_{\mathrm{L} 10}-\Phi_{\mathrm{s} 0}\right)$ is zero because of the same frequency standard. Figure 4 depicts the concept of the calibration on the ground. The phases are measured from $R=R_{1}$ to $R_{2}$, which are known. Thereafter, a black line can be obtained from $R_{1}$ to $R_{2}$. The observed phase at $R$ is

$$
\begin{aligned}
\phi^{i}(R)= & 2 \pi f^{i} \cdot\left(1+r^{i}\right) \cdot(R / c)+2 \pi f^{i} \cdot \tau_{\mathrm{TOTAL}}^{i} \\
& +2 \pi f^{0} \cdot \delta \tau_{0}-2 \pi \cdot N^{i}
\end{aligned}
$$

where, $N^{i}$ is the number denoting cycle ambiguity. If this system were an instrument for measuring range, all values other than the first should be zero. The phase at $R=0$ in the figure would be $2 \pi f^{i} \cdot \tau_{\text {TOTAL }}^{i}+2 \pi f^{0} \delta \tau_{0}$. Then, the measured phase $\phi^{i}(R)$ without $2 \pi$ ambiguity should be

$$
\phi^{i}(R)=2 \pi f^{i} \cdot\left(1+r^{i}\right) \cdot\left(R_{1} / c\right)+2 \pi f^{i} \cdot \tau_{\mathrm{TOTAL}}^{i}+2 \pi f^{0} \cdot \delta \tau_{0}
$$

Since $\delta \tau_{0}$ is known, the $\phi^{i}(R)$ in Eq. (10b) can be obtained at three frequencies, and each of $2 \pi f^{i} \cdot \tau_{\text {TOTAL }}^{i}(i=1,2,3)$ is obtained.

For example, if $R$ is $50 \sim 100 \mathrm{~m}, f^{i}=2.2 \mathrm{GHz}, f^{0}=$ $40 \mathrm{MHz}$, and a phase measurement error is of a few degrees, the $-2 \pi f^{i} \cdot \tau_{\text {TOTAL }}^{i}$ can be estimated with an error of a few degrees from Eq. (10b). The measurements for $i=$ $1,2,3$ are carried out at the same time. The phases at three frequencies are corrected by the observed $-2 \pi f^{i}$. $\tau_{\text {TOTAL }}^{i}-2 \pi f^{0} \delta \tau_{0}+2 \pi f^{0} \cdot \delta \tau_{\mathrm{ST}}$, and the three phases at these frequencies give the correct range.

On the other hand, each lander transmits the signal given by Eq. (3). The phase difference of the transmitted signals from the two landers is given by

$$
\begin{aligned}
\Delta \phi_{\mathrm{L} 2 \mathrm{~B}-\mathrm{L} 1 \mathrm{~B}}= & \phi_{\mathrm{L} 2 \mathrm{~B}}^{i}-\phi_{\mathrm{L} 1 \mathrm{~B}}^{i} \\
= & -2 \pi f^{i} \cdot\left[\left(R_{\mathrm{S}-\mathrm{L} 2}-R_{\mathrm{S}-\mathrm{L} 1}\right) / c\right] \\
& -2 \pi f^{i} \cdot\left(\tau_{\mathrm{L} 2 \mathrm{~F}}^{i}-\tau_{\mathrm{L} 1 \mathrm{~F}}^{i}\right) \\
& -2 \pi\left(f^{i}-f^{0}\right) \cdot\left(\tau_{\mathrm{L} 2 \mathrm{~B}}^{i}-\tau_{\mathrm{L} 1 \mathrm{~B}}^{i}\right) \\
& +2 \pi f^{0} \cdot\left(\tau_{\mathrm{STL} 2}-\tau_{\mathrm{STL} 1}\right) \\
& -\left(\Phi_{\mathrm{L} 20}-\Phi_{\mathrm{L} 10}\right)
\end{aligned}
$$

The first term provides the range difference; the fourth term is obtained from Eq. (8) after determining the range; the second and third terms represent the phases caused by the delay difference in the landers. These terms should be well calibrated. The fourth term is rather small compared with the third one. In particular, the change of the second term with temperature is the main source of error. The characteristics with temperature should be obtained prior to this calculation. 


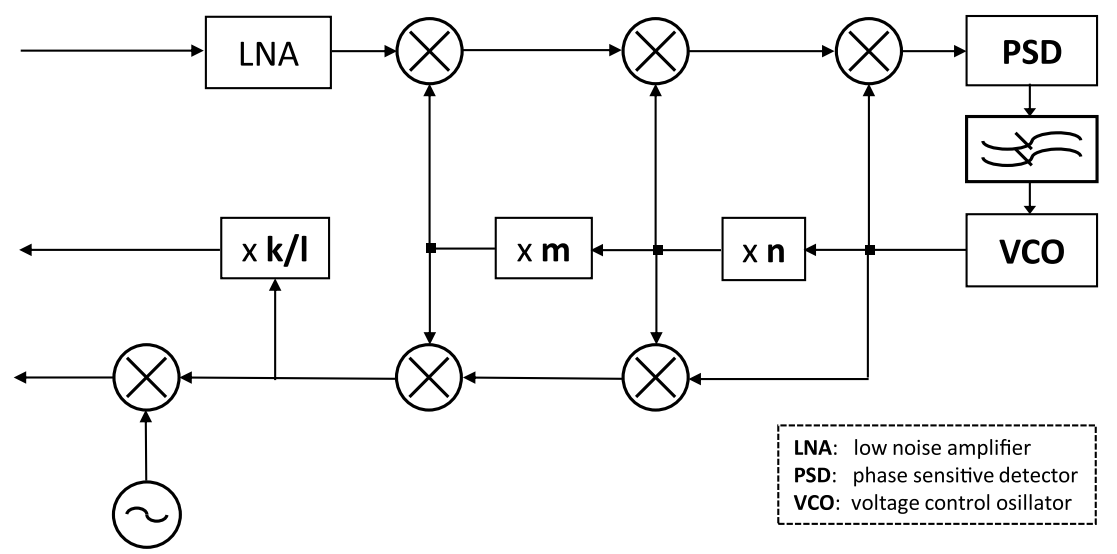

Fig. 5. An example of a roundtrip system with carrier recovery.

Equation (10c) indicates that an original signal is generated at a satellite, and the signal arrives at the Earth through a lander. In order to correct the resultant delay from the satellite to the lander, precise ranging is conducted by the NBV (narrow-band VLBI). From this perspective, a frequency standard in a lander only plays the role of a simple frequency converter. The common signal transmitted from the satellite goes through a lander and arrives at the Earth. The delay (or phase) from the satellite to the lander is estimated separately and is corrected for the total delay of the path from the satellite to the lander.

A measurement test for $2 \pi f^{i} \cdot \tau_{\text {TOTAL }}^{i}$ can be conducted after the calibration of the instrumental delay on the ground by using independent frequency standards. Although the measured phase $\phi^{i}(R)$ varies in this test measurement because $\left(\Phi_{\mathrm{L} 10}-\Phi_{\mathrm{s} 0}\right)$ fluctuates with time, the correct distance $R$ can be estimated after $2 \pi f^{i} \cdot \tau_{\text {TOTAL }}^{i}$ has been corrected by using observed phases at three frequencies.

\subsection{The effect of a phase variation of the frequency standard in the satellite}

The signal $f^{i}$ is generated from the frequency standard of the satellite. The phase noise when $\Delta \phi_{\text {sat }}$ overlaps to the $f^{i}$ signal gives

$$
\Delta \phi_{\mathrm{sat}} \approx 2 \pi f^{i} \cdot \sigma_{y}(\tau) \cdot \tau
$$

where $\sigma_{y}(\tau)$ is the Allan standard deviation. The phase noise is almost cancelled by the correlation of the roundtrip and return signals, but it remains for $\tau$, which is almost equivalent to the roundtrip duration. If we consider the distance between the satellite and the lander to be $2000 \mathrm{~km}$, and $\sigma_{y}(\tau)=10^{-11}$, then the phase noise is estimated from Eq. (11), to be about $0.1^{\circ}$ by taking the roundtrip time as $\tau$.

Since $2 \pi f^{i} \cdot \tau_{\text {TOTAL }}^{i}+\left(\Phi_{\mathrm{L} 10}-\Phi_{\mathrm{s} 0}\right)$ can be determined with an accuracy of about $10^{\circ}$, the accuracy of iVLBI depends on the stability of $2 \pi f^{i} \cdot \tau_{\text {TOTAL }}^{i}$. Particular sources of error are phase changes of RF frequency devices and cables with changes in temperature. Several steps are undertaken in order to minimize these errors. These devices should be installed in a temperature-controlled box. Their characteristics at a specific temperature should be measured carefully on the ground prior to launch. After launch, the temperature should be monitored again. Further calibration can be done for large variations in temperature.

\subsection{Phase difference between the signals received in} the orbiter from the two landers

The phase difference between the signals received from both landers is obtained from Eq. (8) as

$$
\begin{aligned}
\delta \phi_{\mathrm{L} 2 \mathrm{~B}-\mathrm{L} 1 \mathrm{~B}}^{i}= & -2 \pi f^{i} \cdot\left[\left(\tau_{\mathrm{L} 2 \mathrm{~F}}^{i}-\tau_{\mathrm{L} 1 \mathrm{~F}}^{i}\right)+r^{i} \cdot\left(\tau_{\mathrm{L} 2 \mathrm{~B}}^{i}-\tau_{\mathrm{L} 1 \mathrm{~B}}^{i}\right)\right] \\
& +2 \pi f_{\mathrm{SAT}}^{0} \cdot\left(\tau_{\mathrm{STL} 2}-\tau_{\mathrm{STL} 1}\right) \\
& -2 \pi f^{i} \cdot\left(1+r^{i}\right) \cdot\left(R_{\mathrm{S}-\mathrm{L} 2}-R_{\mathrm{S}-\mathrm{L} 1}\right) / c \\
& -\left(\Phi_{\mathrm{L} 20}-\Phi_{\mathrm{L} 10}\right)
\end{aligned}
$$

where $\delta \phi_{\mathrm{L} 2 \mathrm{~B}-\mathrm{L} 1 \mathrm{~B}}^{i}$ is the phase difference between the received signals from the two landers. The second term of Eq. (12) is the delay difference of the cables from the frequency standard to the mixer. This term can be taken as zero as long as the cables are of the same length. Therefore, the cables are to be maintained at same length to the extent possible during manufacturing. The phase difference can be calibrated on the ground, and a marked difference in the phase difference can be corrected. Then, Eq. (12) can be rewritten as

$$
\begin{aligned}
\delta \phi_{\mathrm{L} 2 \mathrm{~B}-\mathrm{L} 1 \mathrm{~B}}^{i}= & -2 \pi f^{i} \cdot\left[\left(\tau_{\mathrm{L} 2 \mathrm{~F}}^{i}-\tau_{\mathrm{L} 1 \mathrm{~F}}^{i}\right)+r^{i} \cdot\left(\tau_{\mathrm{L} 2 \mathrm{~B}}^{i}-\tau_{\mathrm{L} 1 \mathrm{~B}}^{i}\right)\right] \\
& -2 \pi f^{i} \cdot\left(1+r^{i}\right) \cdot\left(R_{\mathrm{S}-\mathrm{L} 2}-R_{\mathrm{S}-\mathrm{L} 1}\right) / c \\
& -\left(\Phi_{\mathrm{L} 20}-\Phi_{\mathrm{L} 10}\right)
\end{aligned}
$$

The effect of the delay and phase instability of the frequency standard in the satellite is cancelled in Eq. (12b). The first term was previously obtained by calibration on the ground. Equation (12b) suggests that the delay changes and phase instability of the frequency standard in the satellite do not directly affect the phase difference of the signals from the two landers.

\subsection{A simple roundtrip system with carrier recovery}

Equations (1) through to (12b) can be applied to the system with a carrier recovery in a lander. This system takes advantage of low power-distribution loss and low transmitting power, but it consists of rather complicated circuits. An example of a roundtrip system with carrier recovery is shown in Fig. 5.

This circuit produces a pure signal that has the same frequency and phase as the signal received by the antenna. 


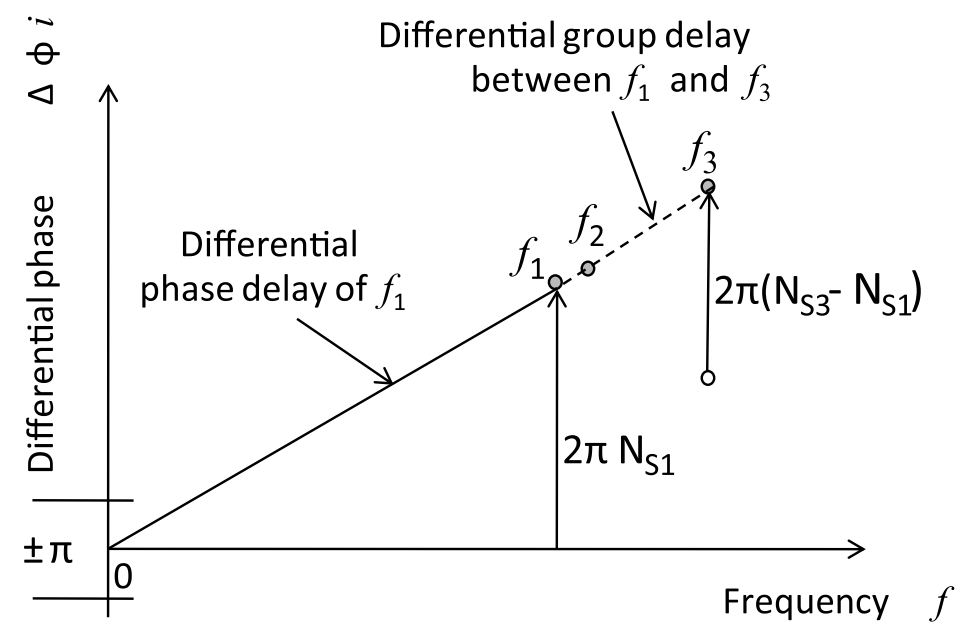

Fig. 6. Principle of the NBV method.

There are two groups of output signals. As shown in Fig. 5, one group of signals is mixed with the standard frequency on the lander. This is called a mixed signal. The other group of signals is the transferred pure signal. The turnaround ratio is given by $k / l$, and this is called a transferred signal. From the transferred signal, the Doppler shift can be obtained on the orbiter with high accuracy. Thereafter, the standard frequency difference of the orbiter and the lander can be obtained from the mixed and transferred signals. In the following, we describe how the phase value caused by the frequency difference can be calculated.

As the standard frequency difference in each observation period can be estimated, the phase caused by the frequency difference can be calculated as

$$
\phi_{\delta f}=2 \pi \cdot \int_{t_{0}}^{t} \delta f_{\mathrm{L} 1 \mathrm{SAT}} d \tau \approx 2 \pi \cdot \sum_{k=1}^{m} \delta f_{\mathrm{L} 1 \mathrm{SAT}} \cdot T_{k}
$$

Where, $T_{k}$ is the time interval between two continuous observation periods.

\section{Delay Determination and an Example of the} Link Budget

4.1 Phase difference between signals received by the antenna on the ground

The phase of the signals received on the ground can be expressed as

$$
\begin{aligned}
\phi_{1}^{i}= & \phi_{\mathrm{L} 1 \mathrm{~B}}^{i}-2 \pi \cdot\left(f^{i}-f_{\mathrm{SAT}}^{0}\right) \cdot\left(r_{1} / c\right) \\
& +2 \pi \cdot \delta f_{\mathrm{L} 1 \mathrm{SAT}} \cdot\left(r_{1} / c\right)-\phi_{\mathrm{trop} 1}-\phi_{\mathrm{iono} 1}^{i}-\phi_{\mathrm{inst} 1}^{i} \\
\phi_{2}^{i}= & \phi_{\mathrm{L} 2 \mathrm{~B}}^{i}-2 \pi \cdot\left(f^{i}-f_{\mathrm{SAT}}^{0}\right) \cdot\left(r_{2} / c\right) \\
& +2 \pi \cdot \delta f_{\mathrm{L} 2 \mathrm{SAT}} \cdot\left(r_{2} / c\right)-\phi_{\mathrm{trop} 2}-\phi_{\mathrm{ino} 2}^{i}-\phi_{\mathrm{ins} 2}^{i}
\end{aligned}
$$

where, $r_{1}$ is the distance from lander 1 to the antenna on the ground station, and $r_{2}$ is the distance from lander 2 to the same antenna. It is also understood that the frequency difference, $\delta f_{\mathrm{L} 1 \mathrm{SAT}}, \delta f_{\mathrm{L} 2 \mathrm{SAT}}$, can be estimated with high accuracy. Further, a rough estimate of the distance between the lander and the antenna can be obtained with an error of several tens of kilometers. The phase caused by the frequency difference between the orbiter and the lander can be estimated with an error that is considerably less than $0.1^{\circ}$. Following the phase correction, the phase difference between the signals received from the two landers can be obtained by

$$
\begin{aligned}
\delta \phi_{2-1}^{i}= & \phi_{2}^{i}-\phi_{1}^{i} \\
= & \left(\phi_{\mathrm{L} 2 \mathrm{~B}}^{i}-\phi_{\mathrm{L} 1 \mathrm{~B}}^{i}\right)-2 \pi \cdot\left(f^{i}-f_{\mathrm{SAT}}^{0}\right) \cdot\left(r_{2}-r_{1}\right) / c \\
& -\left(\phi_{\text {trop2 }}-\phi_{\text {trop } 1}\right)-\left(\phi_{\text {iono2 }}^{i}-\phi_{\text {iono1 }}^{i}\right) \\
& -\left(\phi_{\text {inst } 2}^{i}-\phi_{\text {inst } 1}^{i}\right)
\end{aligned}
$$

The third and fourth terms are almost cancelled to a few degrees because the elongation between two passes is small. These terms are discussed further in Section 6. The fifth term is caused by the phase-frequency characteristic of the antenna, and is sufficiently small. The estimation of the second term is of interest here. It is necessary that the first term be monitored by the orbiter. Substituting Eq. (3) and (12), we obtain,

$$
\begin{aligned}
\delta \phi_{2-1}^{i}= & \left(\phi_{\mathrm{L} 2 \mathrm{~B}}^{i}-\phi_{\mathrm{L} 1 \mathrm{~B}}^{i}\right)-2 \pi \cdot\left(f^{i}-f_{\mathrm{SAT}}^{0}\right) \cdot\left(r_{2}-r_{1}\right) / c \\
= & \delta \phi_{\mathrm{L} 2 \mathrm{~B}-\mathrm{L} 1 \mathrm{~B}}^{i}+2 \pi \cdot\left(f^{i}-f_{\mathrm{SAT}}^{0}\right) \cdot\left(R_{\mathrm{S}-\mathrm{L} 2}-R_{\mathrm{S}-\mathrm{L} 1}\right) / c \\
& -2 \pi \cdot\left(f^{i}-f_{\mathrm{SAT}}^{0}\right) \cdot\left(r_{2}-r_{1}\right) / c+\delta \sigma_{\phi}
\end{aligned}
$$

The second term can be calculated by Eq. (12b). The last term is the result of the phase noise of the frequency standard for the satellite. There exists a time difference between the path from the orbiter-lander 1-antenna and the orbiterlander 2-antenna. The value of this term is miniscule, as can be inferred from Eq. (11); therefore, it can be ignored.

\subsection{Delay determination by narrow-band VLBI}

Three frequencies have been designed in our iVLBI system with the aim of solving the time delay by using a multiple-frequency narrow-band VLBI (NBV) method. The principle of the NBV method is shown in Fig. 6. A detailed description of this process is available in the references listed in Kono et al. (2003) and Kikuchi et al. (2009). In this article, we present the simple process of resolving the ambiguity. The phase at $f_{j}$ is assumed to include the 
Table 1. Ambiguity and resolution of delay.

\begin{tabular}{|l|c|c|c|}
\hline Frequency & $\begin{array}{c}100 \mathrm{KHz} \\
(\mathrm{PRN}: 100 \mathrm{KHz})\end{array}$ & $\begin{array}{c}4 \mathrm{MHz} \\
\left(f_{2}-f_{1}\right)\end{array}$ & $\begin{array}{c}2,280 \mathrm{MHz} \\
\left(f_{3}\right)\end{array}$ \\
\hline Ambiguity of delay & $0.01 \mathrm{~s}$ & $125 \mathrm{~ns}$ & $0.22 \mathrm{~ns}$ \\
\hline Measurement of resolution of phase & - & $4^{\circ}$ (both at two frequencies) & $4^{\circ}$ (both at two frequencies) \\
\hline Resolution of delay & $100 \mathrm{~ns}$ & $\pm 3.93 \mathrm{~ns}$ & $4^{\circ}$ \\
\hline
\end{tabular}

Table 2. Link budget.

\begin{tabular}{|c|c|c|c|c|}
\hline Link stations & & Sat. $\rightarrow$ Lander & Lander $\rightarrow$ Sat. & Lander $\rightarrow$ Earth \\
\hline Frequency & & $2.2 \mathrm{GHz}$ & $2.2 \mathrm{GHz}$ & $2.2 \mathrm{GHz}$ \\
\hline \multicolumn{5}{|l|}{ Transmission } \\
\hline Transmission power & $\mathrm{dBW}$ & 6 & 0 & -3 \\
\hline Feeder loss & $\mathrm{dB}$ & -2 & -2 & -2 \\
\hline Power distribution loss & $\mathrm{dB}$ & 0 & -4.1 & -4.1 \\
\hline Antenna gain & $\mathrm{dBi}$ & -2 & -2 & -2 \\
\hline EIRP & $\mathrm{dBW}$ & 2 & -8.1 & -11.1 \\
\hline Pointing loss & $\mathrm{dB}$ & 0 & 0 & 0 \\
\hline \multicolumn{5}{|l|}{ Propagation } \\
\hline Basic transmission loss & $\mathrm{dB}$ & -168.8 & -168.8 & -210.7 \\
\hline Absorption by atmos. & $\mathrm{dB}$ & 0 & 0 & 0 \\
\hline Absorption by the rain & $\mathrm{dB}$ & 0 & 0 & 0 \\
\hline Polarization loss & $\mathrm{dB}$ & 0 & 0 & 0 \\
\hline \multicolumn{5}{|l|}{ Receiving } \\
\hline Pointing loss & $\mathrm{dB}$ & 0 & 0 & 0 \\
\hline Antenna gain & $\mathrm{dBi}$ & -2 & -2 & 62.1 \\
\hline Feeder loss & $\mathrm{dB}$ & -2 & -2 & -2 \\
\hline Received level & $\mathrm{dBW}$ & -170.8 & -181.0 & -161.7 \\
\hline Tsys & $\mathrm{dBK}$ & 26.8 & 26.8 & 24.6 \\
\hline Noise power density & $\mathrm{dBW}$ & -201.8 & -201.8 & -204.0 \\
\hline Received $C / N_{0}$ & $\mathrm{dBHz}$ & 31.0 & 20.8 & 42.3 \\
\hline $\mathrm{BW}$ & $\mathrm{dBHz}$ & -33 & -10 & -10 \\
\hline$C / N$ & $\mathrm{~dB}$ & -2.0 & 10.8 & 32.3 \\
\hline $\operatorname{Real}[C / N]$ & & 0.6257 & & \\
\hline Power distribution loss & $\mathrm{dB}$ & -4.1 & & \\
\hline RMS phase & deg & & 4.7 & 0.03 \\
\hline
\end{tabular}

cycle ambiguity $N_{\mathrm{s} j}$. First, as shown in Fig. 6, the cycle ambiguity of $N_{\mathrm{S} 2}-N_{\mathrm{S} 1}$ is calculated. Second, the cycle ambiguity of $N_{\mathrm{S} 3}-N_{\mathrm{S} 1}$ is further de ned based on the value of $N_{\mathrm{S} 2}-N_{\mathrm{S} 1}$. Finally, the cycle ambiguity of $N_{\mathrm{S} 1}, N_{\mathrm{S} 2}, N_{\mathrm{S} 3}$ are resolved based on the value of $N_{\mathrm{S} 3}-N_{\mathrm{S} 1}$. When $N_{\mathrm{S} 1}$, $N_{\mathrm{S} 2}, N_{\mathrm{S} 3}$ has been obtained, the differential phase delay for every carrier can be generated.

First, we assigned the frequencies 2,200 MHz (+PRN Code, Chip rate $=100 \mathrm{kHz}), 2,204 \mathrm{MHz}$, and 2,280 MHz in the S-band. These frequencies are applied to resolve the ambiguity. The relationship between ambiguity and resolution of delay is shown in Table 1.

In Table 1, we assume the chip rate of the PRN code to be $100 \mathrm{KHz}$ and the code length to be 1024 bits. The measurement resolution of PRN is $1 / 100$. Therefore, the nal resolution of the time delay can be accurate up to $5 \mathrm{ps}$, which indicates $1.5 \mathrm{~mm}$ in terms of distance.

\subsection{An example of the link budget for a simple roundtrip system with carrier recovery}

Table 2 presents an example of the link budget for the Moon in the simple roundtrip method. The signal received is mixed with a signal from a recovered carrier, and this mixed signal is then transmitted. Given that the noises that are included in the received signal are also ampli ed with the signal, the power-distribution loss reaches $-4.1 \mathrm{~dB}$, as shown here, assuming a bandwidth of $2 \mathrm{kHz}$.

$$
L_{\mathrm{pd}}=(C / N) /(1+C / N)
$$

If we consider that a solar cell produces about several watts $/ 100 \mathrm{~cm}^{2}$ and a lander has dimensions of $30 \times 30 \mathrm{~cm}^{2}$, the power consumption would be limited to several watts. The link budget is satis ed within this limitation.

\section{Design the Positions of the Landers and the Orbit of the Orbiter}

\subsection{Elevation limit}

It is necessary to take into account two conditions when we consider the elevation limit to the observations of a satellite. These constitute an obstacle on the lunar surface as well as to the antenna pattern of a landing unit. If we assume that the unit is not placed very far from the lander, the lander becomes an obstacle after a rover carries the unit. The elevation angle is determined to be about $15^{\circ}$ when the 
unit is $10 \mathrm{~m}$ away from the lander at a height of $2.6 \mathrm{~m}$, and $100 \mathrm{~m}$ away from rocks that are at a height of $26 \mathrm{~m}$.

With regard to the antenna pattern, a patch antenna or a cross-dipole antenna is considered here. A patch antenna with a wide beam width can be designed, for example, $\mp 75^{\circ}$ at about $-6 \mathrm{~dB}$ to the maximum $(+6 \mathrm{~dB})$. The beam width of a cross-dipole antenna has a rather narrow beam width of $\mp 60^{\circ}$ at $-7.4 \mathrm{~dB}$ and $\mp 70^{\circ}$ at $-11 \mathrm{~dB}$ to the maximum $(+2 \mathrm{~dB})$. With these data in mind, the elevation limit of $15^{\circ}$ would be reasonable if a few $\mathrm{dB}$ of the margin in the link budget are known. With regard to the beam width of an antenna onboard an orbiter, the elongation between the two landers is considered to be less than $53^{\circ}$ when the orbital height of an orbiter and the distance between the two landers are both $2,000 \mathrm{~km}$. Therefore, it becomes easy to design an antenna that satis es the condition.

\subsection{Position of a lander}

We consider two landing units on the lunar surface. The following constraints are taken into consideration when designing the position of the lander.

(1) In order to communicate with the Earth, the landers need to be placed on the nearside of the Moon.

(2) To measure the rotation of the Moon, the landers should be placed on the lunar surface along a longitude, to the extent possible.

(3) To measure the libration of the Moon, the landers need to be located on the lunar surface along a latitude-as much as possible. Therefore, the landers are symmetrically placed on the surface about the point $\left(r_{\mathrm{L}}, 0,0\right)$ in the body- xed coordinate, and $r_{\mathrm{L}}$ is the radius of the Moon.

(4) The distance between the two landers needs to be greater than the radius, and it should certainly be less than the diameter of the Moon. If the distance is too large, the improvement in the accuracy of the libration obtained becomes quite limited, but the common view period between two landing units and the orbiter will decrease critically. Therefore, an approximate distance of about 2,000 km is selected as the distance between the two landers.

Considering all of the items shown above, the position of the landers on the lunar surface can be designed as:

Lander 1: longitude $=-30^{\circ}$, latitude $=+25^{\circ}$.

Lander 2: longitude $=+30^{\circ}$, latitude $=-25^{\circ}$.

The distance between the two landers is, then, about $2,154 \mathrm{~km}$.

\subsection{The orbital parameters of the orbiter}

Based on the positions of the two landers shown above, we design the orbit of the orbiter. Our concern was for the common view period between the orbiter and the two landers. When designing the orbit, several rules need be followed:

Rule 1: There exists a common view period during every pass;

Rule 2: The mean value of the common view period should be as large as possible;

Rule 3: The difference of the common view period among the passes should be as small as possible.
By following these rules, we can choose the best orbit.

In terms of the observation of rotation of the Moon, in order to obtain as uniform a common view period as possible, we select the circular orbit, that is, $e=0$. Then, only the semi-major-axis ' $a$ ' (or the height of the orbit) and the inclination ' $i$ ' (relative to the lunar equator) need to be speci ed.

We determine the height of the orbiter now, where:

(1) It is assumed that the half-cone angle of the antenna onboard the orbiter is smaller than $70^{\circ}$. In order to encompass the Moon, the minimal height of the orbiter needs to be greater than $112 \mathrm{~km}$.

(2) It is assumed that the minimal elevation of each antenna onboard the lander is $15^{\circ}$. In order to obtain the common view period, the minimal height of the orbiter should be $h_{\min }=1,070 \mathrm{~km}$. It is important that the common view period lasts for several minutes or longer. The higher orbit will enable this longer common view period to be obtained. Taking into account these conditions, we select the possible height of the orbiter as $1,500,1,800,2,000,2,200$, and 2,500 $\mathrm{km}$ to analyze the ndings.

The inclination of the orbit also needs to be determined. Given the rotation of the Moon, only the scope of $i \in$ $[0,90]^{\circ}$ needs to be considered. Therefore, we consider inclinations of $0^{\circ}, 15^{\circ}, 30^{\circ}, 45^{\circ}, 60^{\circ}, 75^{\circ}$, and $90^{\circ}$.

The results of the simulation can be obtained as follows. The content of each column in Table 3 includes three or four (only when the inclination is equal to zero) data points, which are, sequentially, the number of the common view passes, the mean value of the common view period across all the passes (minutes), the root of covariance of all the common view periods across all the passes (minutes), and the sum of all the common view period (minutes).

As depicted in Table 3, the orbit is in the equatorial plane of the Moon for an inclination that is equal to zero; as such, the common view period is minimally in uenced by the rotation of the Moon. The total number of passes is equal to the number of revolutions, as shown in Fig. 7. If the inclination is greater than $30^{\circ}$, the number of passes of the common view is less than the number of passes, that is, there are some passes with no common view period, as shown in Fig. 8. Considering the rst rule, it is clear that the inclination should be less than $30^{\circ}$. For the condition that $i=15^{\circ}$, the root of the covariance of all common view periods lasts approximately longer than several minutes, and it also does not satisfy the third rule; therefore, we would not prefer this orbit. We consider the $i=0$ to be a better selection for this reason. Since the period varies with the height of the satellite in this condition, the common view period cannot be compared directly. However, the total effective observation time can be calculated. We nd that the common view period increases with increasing orbital height, indicating that a higher orbit will be better suited for our purposes. However, as the height increases, the signal to ratio decreases. Taking all these conditions into consideration, a height $=$ $2,000 \mathrm{~km}$ is optimal. In this case, the common view period is more than $20 \mathrm{~min}$, which is bene cial to the operations on the ground. From the link budget, it is apparent that the 
Table 3. The orbit and common view period.

\begin{tabular}{|c|c|c|c|c|c|}
\hline Inclin.(deg) & 1500 & 1800 & 2000 & 2200 & 2500 \\
\hline 0 & $\begin{array}{l}160 \\
10.14 \\
0.35 \\
1622.4\end{array}$ & $\begin{array}{l}140 \\
18.07 \\
0.26 \\
2529.8\end{array}$ & $\begin{array}{l}129 \\
23.55 \\
0.50 \\
3038.0\end{array}$ & $\begin{array}{l}119 \\
29.20 \\
0.40 \\
3475.8\end{array}$ & $\begin{array}{l}107 \\
37.96 \\
0.19 \\
4061.7\end{array}$ \\
\hline 15 & $\begin{array}{l}160 \\
6.86 \\
3.53\end{array}$ & $\begin{array}{l}140 \\
15.34 \\
4.00\end{array}$ & $\begin{array}{l}129 \\
21.03 \\
4.52\end{array}$ & $\begin{array}{l}119 \\
26.76 \\
5.12\end{array}$ & $\begin{array}{l}107 \\
35.74 \\
5.99\end{array}$ \\
\hline 30 & $\begin{array}{l}54 \\
8.89 \\
3.36\end{array}$ & $\begin{array}{l}64 \\
15.48 \\
9.85\end{array}$ & $\begin{array}{l}69 \\
19.72 \\
12.26\end{array}$ & $\begin{array}{l}71 \\
24.41 \\
14.46\end{array}$ & $\begin{array}{l}75 \\
30.91 \\
18.18\end{array}$ \\
\hline 45 & $\begin{array}{l}34 \\
9.88 \\
9.88\end{array}$ & $\begin{array}{l}41 \\
16.85 \\
14.43\end{array}$ & $\begin{array}{l}44 \\
21.45 \\
17.10\end{array}$ & $\begin{array}{l}43 \\
26.79 \\
20.13\end{array}$ & $\begin{array}{l}47 \\
32.49 \\
24.85\end{array}$ \\
\hline 60 & $\begin{array}{l}29 \\
8.93 \\
7.25\end{array}$ & $\begin{array}{l}34 \\
16.18 \\
11.43\end{array}$ & $\begin{array}{l}35 \\
20.89 \\
13.80\end{array}$ & $\begin{array}{l}37 \\
25.32 \\
16.69\end{array}$ & $\begin{array}{l}36 \\
33.53 \\
20.37\end{array}$ \\
\hline 75 & $\begin{array}{l}29 \\
8.10 \\
4.20\end{array}$ & $\begin{array}{l}33 \\
14.67 \\
6.91\end{array}$ & $\begin{array}{l}35 \\
18.91 \\
8.79\end{array}$ & $\begin{array}{l}35 \\
23.57 \\
10.80\end{array}$ & $\begin{array}{l}35 \\
30.46 \\
13.59\end{array}$ \\
\hline 90 & $\begin{array}{l}32 \\
7.34 \\
3.31\end{array}$ & $\begin{array}{l}37 \\
12.84 \\
5.96\end{array}$ & $\begin{array}{l}37 \\
17.41 \\
7.17\end{array}$ & $\begin{array}{l}36 \\
22.11 \\
8.17\end{array}$ & $\begin{array}{l}37 \\
28.14 \\
11.48\end{array}$ \\
\hline
\end{tabular}

height is feasible. Here, we select $i=0$ and $h=2,000$ $\mathrm{km}$ as examples. The common view period is depicted in Fig. 7.

\section{Time Delay Error Analysis}

\subsection{The phase variation of the system}

From Eqs. (13), (14), and (12b), it is apparent that the error of the time delay between the landers and the antenna is in uenced by the error of $\delta \phi_{2-1}^{i}, \delta \phi_{\mathrm{L} 2 \mathrm{~B}-\mathrm{L} 1 \mathrm{~B}}^{i},\left(\phi_{\text {trop } 2-}\right.$ $\left.\phi_{\text {trop1 } 1}\right),\left(\phi_{\text {iono2 }}^{i}-\phi_{\text {iono1 }}^{i}\right),\left(\phi_{\text {inst2 }}^{i}-\phi_{\text {inst } 1}^{i}\right)$, and $2 \pi\left(f^{i}-f^{0}\right)$. $\left[\left(R_{\mathrm{S}-\mathrm{L} 2}-R_{\mathrm{S}-\mathrm{L} 1}\right) / c\right]$. The $\delta \phi_{2-1}^{i}$ can be obtained on the ground by correlating it with the signals that were received. This error may increase up to $4^{\circ}$. The $\delta \phi_{\mathrm{L} 2 \mathrm{~B}-\mathrm{L} 1 \mathrm{~B}}^{i}$ is obtained on the orbiter and then transmitted to the Earth by telemetry. It is the difference of $\Delta \phi_{\mathrm{PSD}}^{i}$, which is the result of the correlation of the roundtrip, and local return signals on the orbiter. Therefore, this error is considered to be $4 \sqrt{2}^{\circ}$. $2 \pi\left(f^{i}-f^{0}\right) \cdot\left[\left(R_{\mathrm{S}-\mathrm{L} 2}-R_{\mathrm{S}-\mathrm{L} 1}\right) / c\right]$ is caused by the range difference between the two landers and the orbiter. The term is calculated by Eq. (12b). We can consider the error to be $4^{\circ}$.

The two terms of $\left(\phi_{\text {trop2 }}-\phi_{\text {trop } 1}\right)$ and $\left(\phi_{\text {iono2 }}^{i}-\phi_{\text {iono1 }}^{i}\right)$ arise when the signals propagate through the Earth's atmosphere. For a distance of 2,000 km on the lunar surface, the elongation of the signal path on the ground is less than $0.3^{\circ}$. Assuming a same-beam observation and a single-layer screen model of troposphere phase variations in the Kolmogorov turbulence, the travelling time is about $5 \mathrm{~s}$. The phase variation after the signals from the two landers have been cor- related is about $2.4^{\circ}$ (RMS). For the ionosphere, the phase variation includes two components: short- and long-term variations. For the short-term variation, an increase in the integration time can decrease the error. For the long-term variation, the GIM (global ionospheric model) can be used to correct the delay. Because the separation angle between the ground antenna and the two landers is small, the error can be decreased to several millimeters (Liu et al., 2009). If more than one frequency signal can be obtained, for example X-band signal, more accurate results can be derived.

The term of $\left(\phi_{\mathrm{inst2}}^{i}-\phi_{\text {inst1 }}^{i}\right)$ is caused by the nonlinear aspect of the phase-frequency characteristic of the antenna. The phase variation is about $0.2^{\circ}$ in the effective bandwidth of each channel (Liu et al., 2010).

Considering all of these terms, it can be deduced that the total phase variation reaches approximately $13^{\circ}$; that is, the error of the range difference may reach $5 \mathrm{~mm}$. We can also conclude that the error of the time delay is about 20 picoseconds.

\subsection{Required frequency stability of a frequency stan- dard and phase variation with temperature change}

This system needs a stable frequency standard. Since the phase variations caused by the frequency standard of the satellite are nearly cancelled, they should not exceed a few degrees of phase-measurement accuracy during the roundtrip time. Taking the roundtrip time to be less than $10 \mathrm{~ms}$, the phase error $\delta \phi_{\mathrm{st}}$ can be roughly estimated by Eq. (11). Taking $f^{i}$ and $\tau$ as $2.28 \mathrm{GHz}$ and $0.01 \mathrm{~s}$, respectively, the phase variations are small, and $\sigma_{y}(\tau)$ is expected 


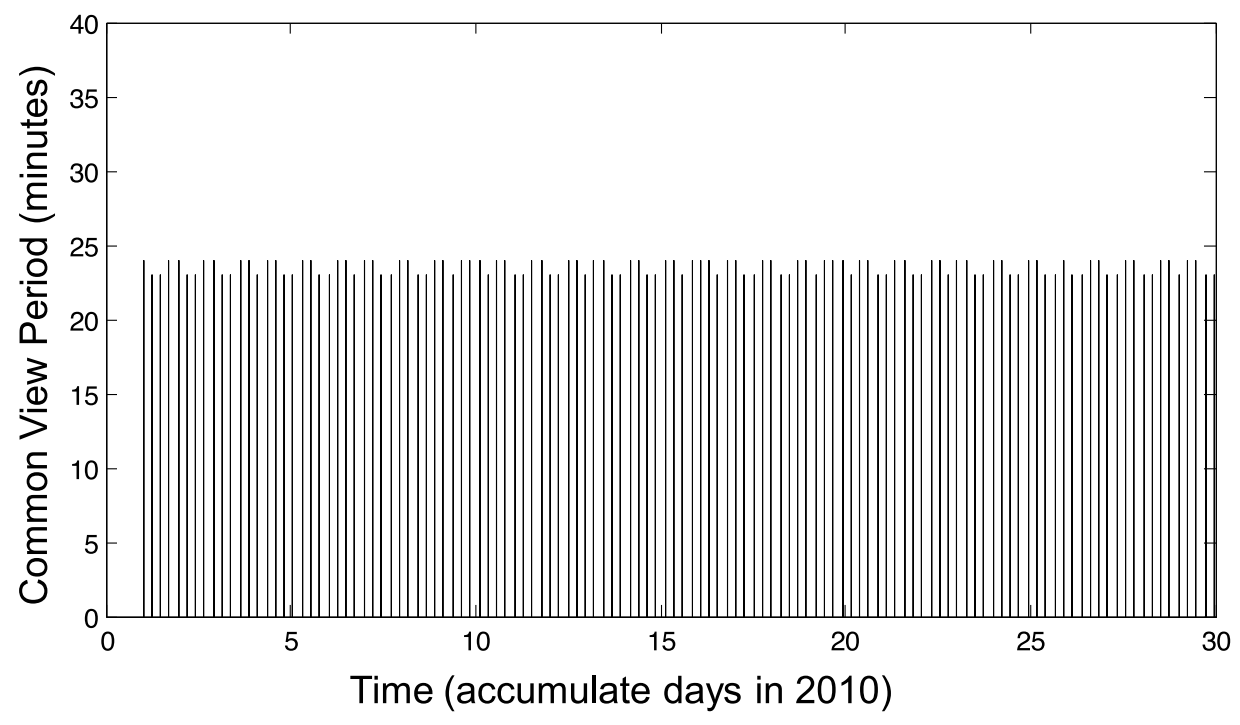

Fig. 7. Common view period $\left(h=2,000 \mathrm{~km}, i=0^{\circ}\right)$.

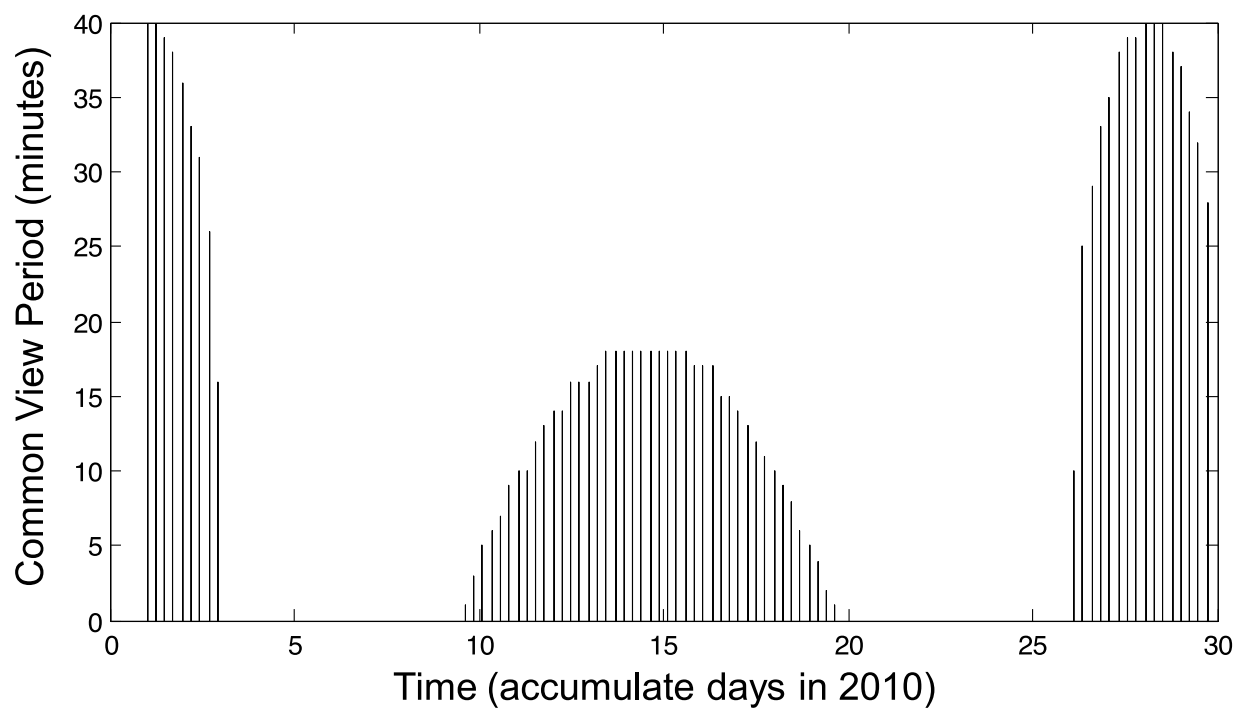

Fig. 8. Common view period $\left(h=2,000 \mathrm{~km}, i=30^{\circ}\right)$.

to be $1 \times 10^{-9}$ at $10 \mathrm{~ms}$. On the other hand, the frequency standard of the lander never yields phase variations that are more than a few degrees during the integration time in order to measure the phase difference with an error of less than a few degrees. If we assume the frequency $f^{i}$ of the standard and integration time $\tau$ in Eq. (11) to be $40 \mathrm{MHz}$ and $10 \mathrm{~s}$, respectively, then $\sigma_{y}(\tau)$ would be $4 \times 10^{-11}$.

The most severe stipulation for frequency standards is the requirement to continuously track the phase difference between the frequency standards of the satellite and the landers. The phase difference is obtained by $-2 \pi \cdot \delta f_{\mathrm{L} 1 \mathrm{SAT}}$. $t-\left(\Phi_{\mathrm{L} 10}-\Phi_{\mathrm{S} 0}\right)$. The phase difference cannot be measured for the interval from one pass to the consecutive pass. The phase change for the interval is to be estimated without $2 \pi$ ambiguity. Since the interval is about $4 \mathrm{~h}$, the $\sigma_{y}(\tau)$ is required to be less than $1 \times 10^{-12}$. The rubidium frequency standard satisfies this condition.

The output frequency and frequency stability of the frequency standard vary with supplied voltage and the temper- ature around the frequency standard. Therefore, delay or phase characteristics of all devices of the system also vary slightly with these parameters. It is inevitable to both stabilize and monitor these parameters.

\section{Lunar Science by the Inverse VLBI}

The inverse VLBI observations provide information about the Earth and the Moon, especially with regard to their rotation. Applying the high-accuracy observation from the iVLBI, the results that are available at present in lunar science can be improved up on. The internal structure and material properties of the Moon are to be deduced from external evidence, and the deepest regions are those that are least understood. The iVLBI provides information with regard to the Moon's tidal response, tidal dissipation, and interactions at the core/mantle interface.

Solid-body tides are raised on the Moon by the gravitational attraction of the Earth. These lunar tidal variations can be represented as sums of periodic components. All of 
the tidal components $>1 \mathrm{~cm}$ occur at about every month or half a month (Williams and Dickey, 2003). These tides can be detected based on the tidal displacements of the landing units.

For the Moon, any description of the three-dimensional rotation requires three Euler angles that can change with time. Two angles describe the orientation of the pole and the orthogonal equator plane, while the third angle generates the rotation about the pole. The angular variations are called physical librations, and the periods of the physical libration terms vary from half a month to almost 273 years (Williams and Dickey, 2003). However, most of these periods last for less than 1 year. Therefore, in order to observe these terms, the lifetime of the iVLBI system should be maintained for more than 1 year.

\section{Conclusion and Discussion}

The conventional VLBI technique or lunar laser ranging has limited application in the ground-based geodetic observation of the Moon, with an error of a few centimeters. The "Inverse VLBI" has been proposed as a breakthrough method to circumvent such limitations. This article describes the system. The most critical aspect of the technique is how to obtain the phase difference measurements of the two landers. We propose a simple roundtrip signal method to obtain this phase difference. Then, the calibration of the instrumental delay on the ground is provided. The time delay is determined by the NBV method, and an example of the link budget is shown. We also report on the design of the position of the landers and the orbit of the orbiter based on the necessity of the observation, and the common view period is analyzed. After analyzing this error, we conclude that the range difference can be obtained with an error of several millimeters. Hence, the iVLBI technique can achieve geodetic observation with only an error of a few millimeters, while the error of geodetic observation in the conventional VLBI will be magni ed with the distance between object and stations. In order to realize the iVLBI system, new equipment for implementing the iVLBI should be developed. In addition, testing on the ground should be carried out prior to launch. Phase noise changes with temperature should also be carefully researched for this method.

We hope that a series of new results can be obtained by using the iVLBI technology. Simulation analyses on solving the parameters of the lunar rotation are expected to be executed in the near future. It is also apparent that the suitable range can be obtained after calibrating the time delay of the landers.

This method can be applied to observe the rotation of the Mars as well as other planets. It is in uenced very minimally by the distance between the planet and the Earth. If the planet is farther away from the Earth, elongation of the two signal passes received on the ground will be very minimal, and the in uence of the Earth's atmosphere will be decreased to a very low level. More high-accuracy observations can be facilitated under such conditions.

Acknowledgments. This work was supported by the programs of Chinese Academy of Sciences for Visiting Professorship for Senior International Scientists, Hundred Talent Project of Chinese Academy of Sciences, National Natural Science Foundation of China (Grant Nos. 10973031 and 11043004), and the RISE/SELENE Project of the National Astronomical Observatory of Japan.

\section{References}

Kawano, N., M. Hosokawa, H. Hanada, and M. Imae, Inverse VLBI Method for Planetodesy, J. Geod. Soc. Jpn., 45(3), 181-203, 1999.

Kawano, N., Q. Liu, J. Ping, T. Iwata, and F. Kikuchi, Inverse VLBI by various techniques for planetodesy, IEICE Technical Report SANE2009105, 2009.

Kawano, N., M. Chen, J. Sun, and K. Shang, Inverse VLBI system and planetodesy, New advances in lunar exploration, Proceedings of International Symposium on Lunar Science (ISLS2010), 149-156, 2010.

Kikuchi, F., Q. liu, H. Hanada, N. Kawano, K. Matsumoto, T. Iwata, S. Goosens, K. Asari, Y. Ishihara, S. Tsuruta, T. Ishikawa, H. Noda, N. Namiki, N. Petrova, Y. Harada, J. Ping, and S. Sasaki, Picosecond accuracy VLBI of the two sub-satellites of SELENE(KAGUYA) using multi-frequency and same beam methods, Radio Sci., 44, 68-73, 2009.

Kono, Y., H. Hanada, J. Ping et al., Precise positioning of spacecraft by multi-frequency VLBI, Earth Planets Space, 55, 581-589, 2003.

Liu, Q., X. Shi, F. Kikuchi et al., High-accuracy same-beam VLBI observations using Shanghai and Urumqi telescopes, Sci. China Ser G-Phys Mech. Astron., 52(12), 1858-1866, doi:10.1007/s11433-009-0280-1, 2009.

Liu, Q., M. Chen, W. Xiong et al., Relative position determination of a lunar rover by using high-accuracy multi-frequency same-beam VLBI, Sci. China Ser. G, 53(3), 1-8, 2010.

Takahashi, Y., F. Nakagawa, H. Kunimori, J. Amagai, S. Tsuchiya, R. Tabuchi, and S. Hama, Precise time transfer and ranging experiment result between ground and ETS-VIII, Proceedings of International Symposium on GPS/GNSS 2008, 659-663, 2008.

Williams, J. G. and J. O. Dickey, Lunar geophysics, geodesy, and dynamics, in 13th International Workshop on Laser Ranging, edited by Noomen, R., S. Klosko, C. Noll, and M. Pearlman, NASA/CP-2003212248, 75-86, 2003.

M. Chen (e-mail: chenming@shao.ac.cn), N. Kawano, K. Shang, J. Sun, Q. Liu, F. Kikuchi, and J. Ping 